\title{
A Wireless Sensor Network Solution for Precision Agriculture Based on ZigBee Technology
}

\author{
Manijeh Keshtgari ${ }^{1}$, Amene Deljoo ${ }^{2}$ \\ ${ }^{1}$ Department of Computer Engineering \& IT, Shiraz University, Shiraz, Iran \\ ${ }^{2}$ Department of E-Learning, Shiraz University, Shiraz, Iran \\ Email: amene.deljoo@gmail.com
}

Received October 13, 2011; revised November 20, 2011; accepted December 23, 2011

\begin{abstract}
Wireless Sensor Networks (WSNs) have attracted much attention in recent years. The potential applications of WSNs are immense. They are used for collecting, storing and sharing sensed data. WSNs have been used for various applications including habitat monitoring, agriculture, nuclear reactor control, security and tactical surveillance. The WSN system developed in this paper is for use in precision agriculture applications, where real time data of climatologically and other environmental properties are sensed and control decisions are taken based on it to modify them. The architecture of a WSN system comprises of a set of sensor nodes and a base station that communicate with each other and gather local information to make global decisions about the physical environment. The sensor network is based on the IEEE 802.15.4 standard and two topologies for this application.
\end{abstract}

Keywords: Wireless Sensor Network; Precision Agriculture; Grid Topology; ZigBee Network

\section{Introduction}

Precision agriculture can be defined as the art and science of using advanced technology to enhance crop production. Wireless sensor network is a major technology that drives the development of precision agriculture. The science and engineering questions associated with precision agriculture center around increasing the efficiency to prosper in a sustainable manner. Increases in agricultural efficiency will stem from networking sensors to elucidate important spatiotemporal patterns and integrating their data streams so as to not only display or record information, but to actuate human and autonomous responses. The concept of precision agriculture has been around for some time now. Blackmore et al., in 1994 [1] defined it as a comprehensive system designed to optimize agricultural production by carefully tailoring soil and crop management to correspond to the unique condition found in each field while maintaining environmental quality. In California, Beckwith et al designed deployed and analyzed output of a large scale implementation of a wireless sensor network in a vineyard [2]. 65 motes with a maximum of 8 hops were deployed in a planned area where no neighbor discovery features were implemented and a table driven protocol was used rather than a self organizing network. Data was recorded every five minutes with a grid of sensor nodes each separated $15 \mathrm{~m}$ from the other. In Europe, the Lofar Agro project is a study of precision agriculture that focuses on tailored management of a crop. This involves monitoring soil, crop and climate conditions in a field, generalizing the result and providing a decision support system (DSS) for treatments or taking differential action such as real time variation of fertilizer or pesticide application [3].

The objective of this paper is to report the design, construction, and testing of a distributed infield WSN, a remote monitoring control, grid topologies.

This article is organized in the following way: In Section 2, we present the system requirement and architecture in precision agriculture; in Section 3, we have a brief overview of wireless standard and appropriate wireless technology; in Section 4, we identify the proposed methodology for this work; Section 5 presents the system model; In Section 6, we discuss the results and challenges in the development of the application and, finally, Section 7 conclude the paper.

\section{System Requirement and Architecture}

The requirements that adopting a WSN are expected to satisfy in effective agricultural monitoring concern both system level issues (i.e., unattended operation, maximum network life time, adaptability or even functionality and protocol self-reconfigurability) and final user needs (i.e., communication reliability and robustness, user friendlyness, versatile and powerful graphical user interfaces). 
The most relevant mainly concerns the supply of standalone operations. To this end, the system must be able to run unattended for a long period, as nodes are expected to be deployed in zones that are difficult to maintain [4-5]. This calls for optimal energy management ensuring that the energy spent is directly related to the amount of traffic handled and not to the overall working time. In fact, energy is nevertheless a limited resource and the failure of a node may compromise WSN connectivity as the network gets partitioned. Other issues to be addressed are the capabilities of quickly setting-up an end-to-end communication infrastructure, supporting both synchronous and asynchronous queries, and of dynamically reconfiguring it. Other important properties are scalability and adaptability of the network's topology, in terms of the number of nodes and their density in unexpected events with a higher degree of responsiveness and reconfigurability. This also implies the development of a plug and play sensor interface and the provisioning of remote connectivity. Finally, several user-oriented attributes, including load, latency and throughput are calculated. The system, shown in Figure 1, comprises a self-organizing WSN endowed with sensing capabilities, a ZigBee Gateway, which gathers data and send to the base station [6].

\section{Wireless Standards and Proprietary Wireless Sensor Technologies}

There exist four task groups within the 802.15 working group. Task group one (802.15.1) defined a standard for WPANs based on the physical (PHY) and MAC layers of the Blue-tooth specification version 1.1 [7]. Task group two (802.15.2) is developing a model for the coexistence of WLAN (802.11) and WPAN (802.15). Task group three (802.15.3) is developing standards for high data rate WPANs (20 Mbps and greater). Task group four (802.15.4) is responsible for developing $\mathrm{PHY}$ and MAC layer standards for low data rate and low complexity solutions.

The IEEE 802.15.4 standard is a physical radio specification providing for low data rate connectivity among relatively simple devices that consume minimal power and typically connect over short distances [8]. It is ideal for monitoring, control, automation, sensing and tracking applications for the home, medical and industrial environments. ZigBee is a wireless networking standard that is aimed at remote control and sensor applications which is suitable for operation in harsh radio environments and in isolated locations.

The ZigBee standard supports three device types: ZigBee Coordinator, ZigBee Router, and ZigBee End Device [9-11]. Each device type implements varying levels of functionality with associated cost impacts. Thus, equipment manufacturers and system developers may implement network topology and tradeoff functionality with overall cost.

The Application Support Sub layer (APS) provides a control interface to communicate with the application as well as maintaining up-to-date binding tables.

ZigBee is established by the ZigBee Alliance that is supported by more than 70 member companies. It adds network, security and application software to the IEEE 802.15.4 standard. Owing to its low power consumption and simple networking configuration, ZigBee is considered the most promising for wireless sensors. Currently, the ZigBee specification is still under development. Table 1 compares the three wireless standards that are most suitable for wireless. As it shown in the Table 1 ZigBee is more suitable for our application.

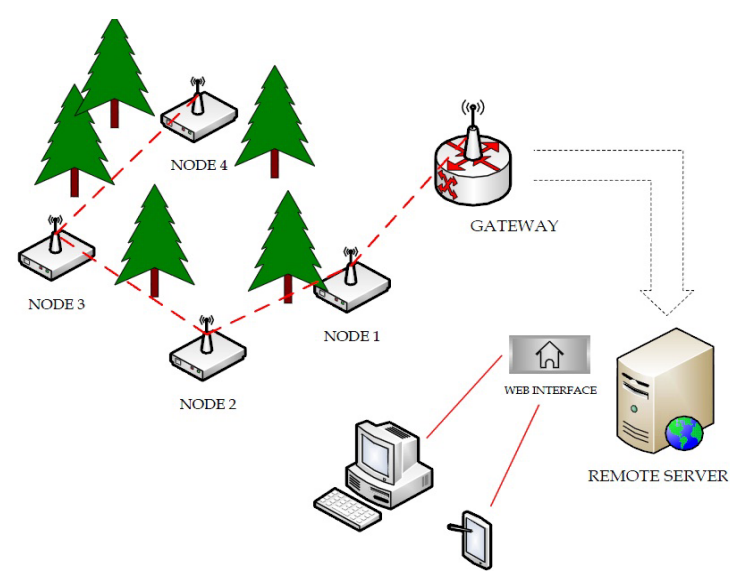

Figure 1. WSN schema.

Table 1. Comparison between wireless LAN, bluetooth and ZigBee.

\begin{tabular}{|c|c|c|c|}
\hline Feature & Wi-Fi (IEEE 802.11 b) & Bluetooth(IEEE 802.15.1) & ZigBee (IEEE 802.15.4) \\
\hline Radio & $\mathrm{DSSS}^{1}$ & FHSS $^{2}$ & DSSS \\
\hline Data rate & $11 \mathrm{Mbps}$ & $1 \mathrm{Mbps}$ & $250 \mathrm{kbps}$ \\
\hline Slave enumeration latency & 32 & 7 & 64,000 \\
\hline Node per master & Up to $3 \mathrm{~s}$ & Up to $10 \mathrm{~s}$ & $30 \mathrm{~ms}$ \\
\hline Data type & Video, audio, graphics,pictures,files & audio, graphics, pictures, files & Small data packet \\
\hline Range (m) & 100 & 10 & 70 \\
\hline Extend ability & Roaming possible & No & Yes \\
\hline Battery life & Hours & 1week & >1year \\
\hline Bill of material (\$) & 9 & 6 & 3 \\
\hline Complexity & complex & Very complex & simple \\
\hline
\end{tabular}

1. direct sequence spread spectrum; 2 . frequency hopping spread spectrum. 


\section{Sensor Network, the Propose Methodology}

In this paper, we propose two topologies for precision agriculture. In the first one, each sensor node is placed at the corner of each grid and server node is located in the middle of area. In the second scenario, server nodes are placed at out of area. Finally, we compare two models and choose the appropriate topology. The topology should guarantee QoS while taking into consideration the limited power and optimization of communication.

The aforesaid approach is practical for the separation of field in management also, monitoring areas, in order to avoid the node placement in grid placement. An appropriate number of sensors which will completely cover the areas completely.Therefore, the aspect of data are placed so that a complete picture of the total field is received. For our proposed model, we used 24 WSN under ZigBee network with specific properties. The properties are shown in Table 2. Moreover, we illustrated properties antenna which we use in Table 3.

Simulations are done using the two following network topologies as shown in Figures 2 and $\mathbf{3}$.

- A grid topology of 24 sensors with $16 \mathrm{~m}$ vertical and horizontal distance each. Moreover, we use 1 Gateway that is played role as sink node. Sink node is placed on mid road of the farm as shown in Figure 2. In this model sensor nodes which are on the left side of the road sense agriculture parameters and forward them to the sink node.

First, each of sensors communicates with sink node through wireless network (as we mentioned before ZigBee network). After that, sink node should transfer real time data to the base station

- Another scenario of 24 sensors that is distribution in the felid which the server node is placed out of area as shown in Figure 3. It is called proposed topology.

The coverage distance for each node depends on the type of node and RF technology. The nodes were not put at the maximum coverage distance for more power efficiency. The medium distance of $10 \mathrm{~m}$ results from distances of sensors, in which there is a high possibility of communication between them (the Signal-to-Noise ratio was above a certain threshold).

\section{System Model}

Since a variety of topologies for wireless sensor network has been developed and each topology has its own scenario, it is hard to compare all of these topologies. We use OPNET to deal with the challenges of comparing different topologies.

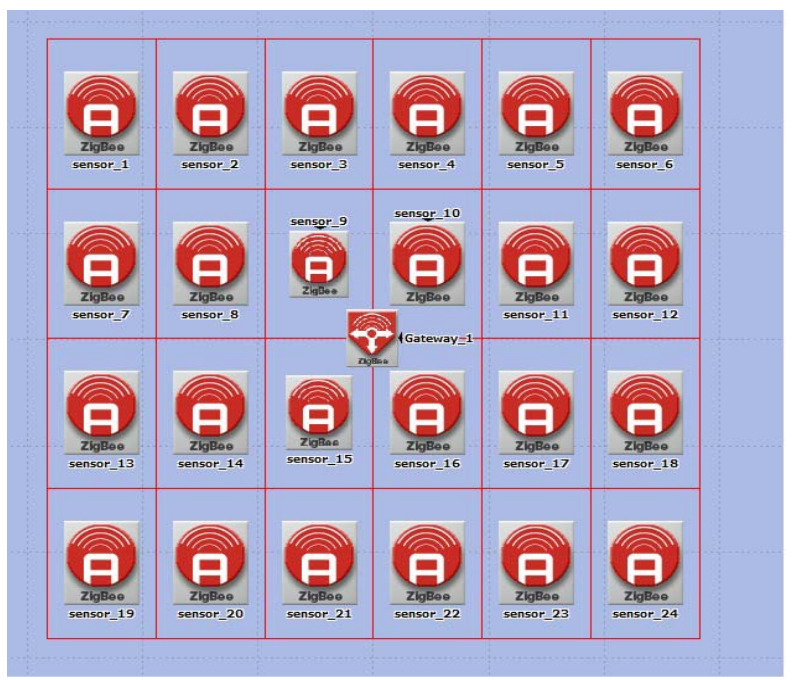

Figure 2. Grid topology.

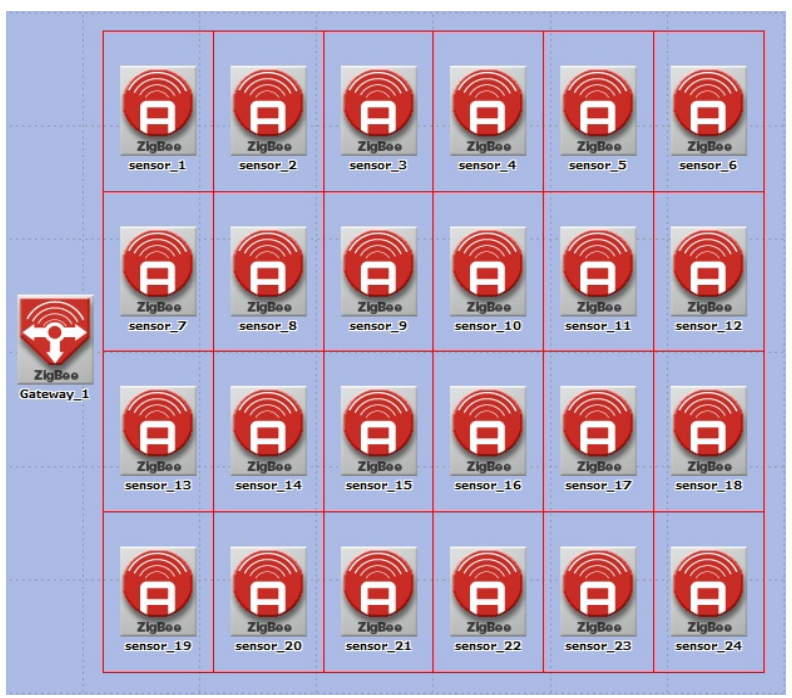

Figure 3. Grid topology (scenario 2).

Table 2. Sensor properties.

\begin{tabular}{lccccccc}
\hline \multirow{2}{*}{ SENSOR SPECIFICATION } & Model & Protocol & Frequency & Tx power & Sensitivity & Range \\
\cline { 2 - 7 } & ZigBee & IEEE 802.15 .4 & $2.4 \mathrm{GHZ}$ & $1 \mathrm{mw}$ & $-92 \mathrm{dbm}$ & $500 \mathrm{~m}$ \\
\hline
\end{tabular}

Table 3. Antenna Properties.

\begin{tabular}{llll}
\hline & Dimensions & Gain & Type \\
\cline { 2 - 4 } ANTENNA SPECIFICATION & $224 \times 22 \mathrm{~mm}$ & $5 \mathrm{dbi}$ & Omni directional \\
\hline
\end{tabular}


In our research, Gateway is placed on the middle of each cell of the farm zone as shown in Figure 2 for grid topology. In this model, sensor nodes which are on the left and right side of the road will sense agriculture parameters and forward those to the base station every 15 minute, which are indicated by red arrows in Figure 2. Therefore, we have 24 sensors with $16 \mathrm{~m}$ of vertical and horizontal distance between them.

We use different range of IP addresses for connecting sensors to the base station. Hence, monitoring system with this feature is controlled and managed each sensor area with a specific function. Also, in the base station, end-to-end delay for each node is monitored.

We have another scenario which is Grid topology. It has the same setting like the previous model. In this model, sink node (gateway) is located out of area as shown in Figure 3.

Here, for both topologies, we consider the same technical setting for sensors which are shown in Tables 2 and 3.

\section{Simulation Reasult}

Propos we consider a set of performance metrics for comparing different topologies, including delay, throughhput, and load. The simulation time is 50 minutes. Some definitions of the metrics are:

- Throughput: Number of messages are received per second. The throughput of the network is the sum of the throughputs of all the destinations.

- Delay: Time to send a message from source to destination. For any destination, if $\mathrm{n}$ packets have arrived, delay for that destination is given by Equation 1 . Where di is the delay of the ith packet. Network delay is. Averaged by the number of destinations.

$$
\text { Delay }=\sum_{i=1}^{n} \frac{\mathrm{d} i}{n}
$$

- Load: Number of packets that can be sent by the network at one time.

Delay in grid case is much regular than delay in second scenario as shown in Figure 4. We have calculated the average network delay using the simulation for the two cases. It is $0.018 \mathrm{sec}$ for the grid and 0.010 for the second topology. In second topology, we observed that after 20 minutes we have no signal, it means that most of the packet are dropped.

Proposed topology throughput is lower than that in grid as shown in Figure 5, because in grid topology the total packets sent to the destination (implying that we consider $100 \%$ delivery rate) are much more than the packets sent by sources in the proposed topology.

In grid topology, load for a long portion of simulation time is stable as is shown in Figure 6. This means that more packets are likely to be delivered to the destination through the network. Approximately after 50 minute in grid topology, the load is near $89 \%$. On the other hand, in proposed topology, we observe that not only load is on average constant, but it has stopped after 20 minute, the packets are dropped and load is approximately $35 \%$.

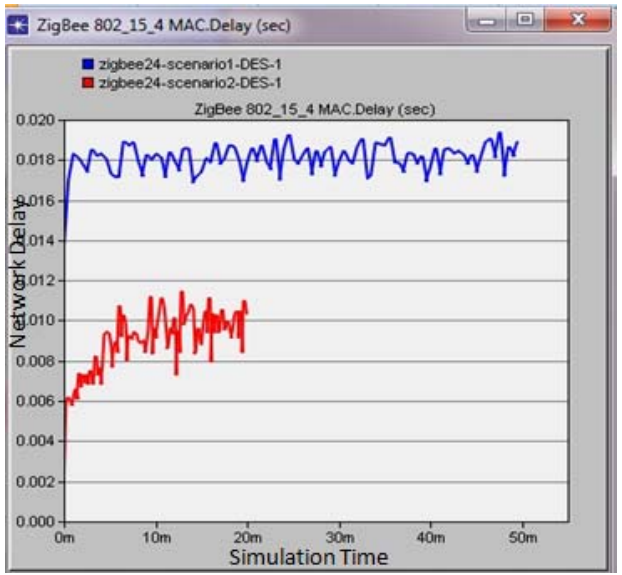

Figure 4. Both network delay.

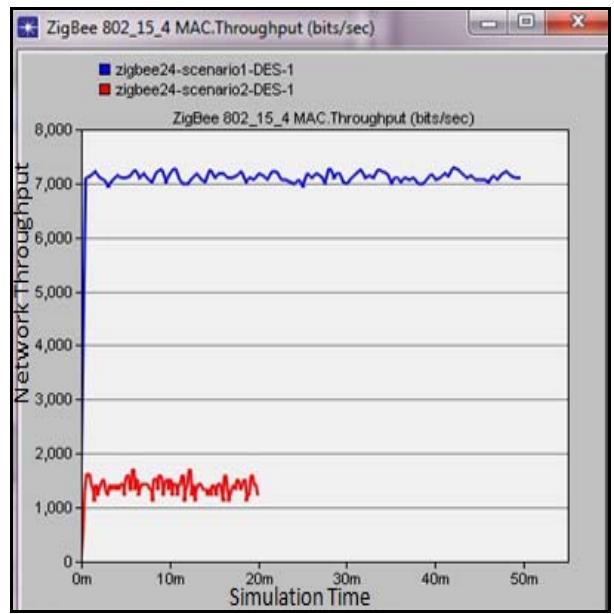

Figure 5. Both network throughput.

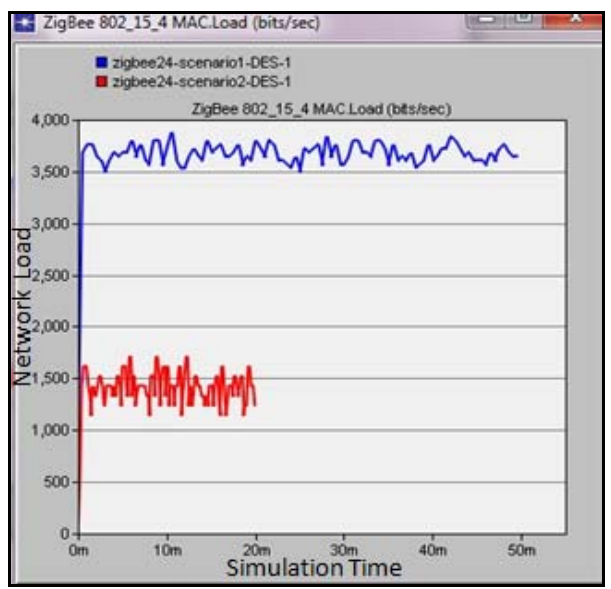

Figure 6. Both network load. 


\section{Conclusions}

Precision agriculture and WSN applications combine an exciting new area of research that will greatly improve quality in agricultural production, precision irrigation and will have dramatic reduction in cost needed. Furthermore, the ease of deployment and system maintenance, monitoring opens the way for the acceptation of WSN systems in precision agriculture. Using the proposed methodology, in finding the optimal sensor topology, we contrive to lower implementation cost and thus make WSN a more appealing solution for all kinds of fields and cultivations.

This paper proved potential applications of ZigBee wireless technology in agricultural systems can be extended to real time field monitoring, automated irrigation control, monitoring, and remote operation of field machinery.

\section{REFERENCES}

[1] S. Blackmore, "Precision Farming: An Introduction," Outlook on Agriculture Journal, Vol. 23, 1994, pp. 275280.

[2] N. Wang, N. Zhang and M. Wang, "Wireless Sensors in Agriculture and Food Industry: Recent Development and Future Perspective," Computers and Electronics in Agriculture Journal, Vol. 50, 2006, pp. 114-120.

[3] I. F. Akyildiz and W. Xudong, "A Survey on Wireless Mesh Networks,” IEEE Communication Magazine, Vol. 43, 2005, pp. S23-S30. doi:10.1109/MCOM.2005.1509968

[4] J. Al-Karaki and A. Kamal, "Routing Techniques in Wireless Sensor Networks: A Survey,” IEEE Communication Magazine, Vol. 11, 2004, pp. 6-28. doi:10.1109/MWC.2004.1368893

[5] K. Langendoen and G. Halkes, "Energy-Efficient Medium Access Control," The Embedded Systems Handbook, Vol. 4, No. 3, 2004, pp. 2-30.

[6] A. Pennacchia, P. Mazzolai and P. Dario, "Development of a Programmable Sensor Interface for Wireless Network Nodes for Intelligent Agricultural Applications,” Proceedings of IE, IEEE Computer and Communications Societies, Sydney, 2005, pp. 1-6.

[7] M. Sveda, P. Benes, R. Vrba and F. Zezulka, "Introduction to Industrial Sensor Networking," Handbook of Sensor Networks: Compact Wireless and Wired Sensing Systems, 2005, pp. 10-24.

[8] A. El-Hoiydi, J. Decotignie, C. Enz and E. Le Roux, "WiseMAC, an Ultra Low Power MAC Protocol for the WiseNET Wireless Sensor Network," Proceedings of SENSYS, Association for Computer Machinery, Los Angeles, 2003, pp. 244-251.

[9] W. Ye, J. Heidemann and D. Estrin, “An Energy-Efficient MAC Protocol for Wireless Sensor Networks,” Proceedings of INFOCOM, IEEE Computer and Communications Societies, New York, 2002, pp. 1567-1576.

[10] T. Dam and K. Langendoen, “An Adaptive Energy-Effi- cient MAC Protocol for Wireless Sensor Networks,” Proceedings of SENSYS, Association for Computer Machinery, Los Angeles, 2003, pp. 171-180.

[11] G. Lu, B. Krishnamachari and C. Raghavendra, “Adaptive Energy-Efficient and Low-Latency MAC for Data Gathering in Sensor Networks," Proceedings of WMAN, Institute fur Medien Informatik, Ulm, 2004, pp. 24402443.

[12] S. Shakkottai, T. Rappaport and P. Karlsson, "CrossLayer Design forWireless Networks,” IEEE Communication Magazine, Vol. 41, 2003, pp. 77-80. doi:10.1109/MCOM.2003.1235598

[13] Y. J .Kim, R. G. Evans and W. M, "Remote Sensing and Control of an Irrigation System Using a Distributed Wireless Sensor Network," Proceedings of IEEE Symptoms, IEEE Transaction on Instrumentation and Measurement, Florida, Vol. 57, No. 7, 2008, pp. 1379-1387.

[14] G. H. E. L. Lima, L. C. Silva and P. F. R. Neto, "WSN as a Tool for Supporting Agriculture in the Precision Irrigation,” International Conference on Nitride Semiconductors, 2010, pp. 137-142.

[15] Y. Xijun, L. Limei and X. Lizhong, “The Application of Wireless Sensor Network in the Irrigation Area Automatic System,” Networks Security, Wireless Communications and Trusted Computing, Vol. 1, 2009, pp. 21-24. doi:10.1109/NSWCTC.2009.118

[16] M. A. M. Vieira, C. N. Coelho, D. C. Jr. Silva and J. M. Mata, "Survey on Wireless Sensor Network Devices," IEEE Conference on Emerging Technologies and Factory Automation Proceedings, Vol. 1, 2003, pp. 537-544.

[17] H. Karl and A. Willig, "Protocols and Architectures for Wireless Sensor Networks," John Wiley \& Sons, New York, 2005. doi:10.1002/0470095121

[18] S. Yi, "The Short-Distance Wireless Communication and Network Technology,” National Academies Press, Washington D.C, Vol. 2, 2009.

[19] R. W. Wall and B. A. King, "Incorporating Plug and Play Technology into Measurement and Control Systems for Irrigation Management," Presented at the ASAE/CSAE Annual International Meeting, Ottawa, 2004, pp. 42-189.

[20] T. Wark, P. Corke, P. Sikka and L. Klingbeil, "Transforming Agriculture through Pervasive Wireless Sensor Networks,” Pervasive Computing IEEE, Vol. 6, No. 2, 2007, pp. 50-57. doi:10.1109/MPRV.2007.47

[21] B. Han, "The Principle and Network Optimizing of GPRS,”Vehicular Technology Conference Proceedings, Tokyo, Vol. 1, 2004, pp. 440-444.

[22] K. Konstantinos, X. Apostolos, K. Panagiotis and S. George, "Topology Optimization in Wireless Sensor Networks for Precision Agriculture Applications,” Sensor Communication, 2007, pp. 526-530.

[23] A. Baggio, "Wireless Sensor Networks in Precision Agriculture,” Delft University of Technology, Delft, 2009.

[24] W. Cao, G. Xu, E. Yaprak, R. Lockhart, T. Yang and Y. Gao, "Using Wireless Sensor Networking (WSN) to Manage Micro-Climate in Greenhouse,” Manufacturing Enterprise Solutions Association, Vol. 17, No. 3, 2008, pp. 636-641. 
[25] W. Zhang, G. Kantor and S. Singh, "Integrated Wireless Sensor/Actuator Networks in Agricultural Applications,”
In 2nd ACM International Conference on Embedded Networked Sensor Systems, 2004, p. 317. 